\section{Chronic prostatitis and pelvic pain: role of anti-nanobacterial therapy}

Chronic prostatitis/chronic pelvic pain syndrome (CPPS) is a common problem and is often refractory to treatment. Prostatic calcifications have been implicated in the etiology of this condition, although this idea is contentious. Because the core of prostatic calculi contain apatite-a mineral formed by nanobacteria-researchers from the Cleveland Clinic in Florida asked whether the elimination of nanobacterial calcification might resolve the symptoms of CPPS.

Shoskes et al. tested their hypothesis in 16 men with recalcitrant CPPS of more than 9 months duration. All had failed antibiotic and anti-inflammatory drug therapy and had prostatic calculi on transrectal ultrasound. Patients were treated daily with comET (Nanobac Life Sciences, Tampa, FL), which has three components: $500 \mathrm{mg}$ tetracycline to eradicate the nanobacteria; a nutritional supplement thought to allow the antibiotic to penetrate the stone; and an ethylenediamine tetraacetic acid suppository to break down the stone.

After 3 months' treatment, symptoms had improved significantly: the mean total score using the NIH Chronic Prostatitis Symptom Index had fallen to $13.7 \pm 2.0$, compared with $25.7 \pm 1.6$ before treatment $(P<0.0001)$. An 'extremely significant' $50 \%$ improvement in symptoms was seen in eight (53\%) of the evaluable patients. The minimal side effects included fatigue and rectal discomfort.

The authors note that the observed improvements in symptoms could be attributed to any or all of comET's three components, and that the placebo effect could not be ruled out. They call for prospective, placebo-controlled trials to further investigate whether this treatment is useful in treating the symptoms of CPPS.

Original article Shoskes DA et al. (2005) Anti-nanobacterial therapy for men with chronic prostatitis/chronic pelvic pain syndrome and prostatic stones: preliminary experience.

J Urol 173: 474-477

\section{Genital ambiguity and urogenital sinus anomalies: a new classification}

Since the 1950s, pediatric urologists have used Prader's classification to describe cases of genital ambiguity, and have generally characterized urogenital sinus anomalies based on 'high' or 'low' urogenital confluence. Rink and colleagues have recently proposed a new system-termed PVE classification-which aims to provide more accurate descriptions of these highly variable abnormalities.

In the PVE system, 'P' documents the maximal length of the stretched phallus in its flaccid state, and phallic width measured ventrally from side to side. For example, ' $P_{3,1}$ ' would describe a case where the phallus is $3 \mathrm{~cm}$ long and $1 \mathrm{~cm}$ wide. Next, ' $V$ ' denotes the distance, in $\mathrm{cm}$, from the vaginal confluence to the bladder neck and to the perineal meatus. These two measurements are made endoscopically, using a catheter as a guide. Finally, 'E' refers to the external genital appearance. This is based on the Prader numbers $1-5$, with the addition of class ' 0 ' to represent normal anatomy, and class ' 6 ' to denote rare deviations that fall outside the other categories (for example, the blank or 'doll-like' perineum).

In summary, the PVE classification describes the phallic size, true location of the vaginal confluence, and the external genital appearance. Rink et al. hope that the system will be useful in the surgical management of genital ambiguity and urogenital sinus anomalies, and will allow more meaningful comparisons of surgical outcomes.

Original article Rink RC et al. (2005) A new classification for genital ambiguity and urogenital sinus anomalies. BJU Int 95: $638-642$

\section{Urinary incontinence: results of a large survey of US women}

Results from a large postal survey in the US have indicated a high prevalence of urinary incontinence among women. The condition was reported by $42 \%$ of respondentsgenerating an estimated population-based prevalence of $45 \%$ after adjusting for oversampling of younger women-and $18 \%$ were severely affected. Furthermore, the study revealed that the problem is not restricted to the elderly, because over a quarter of women aged 30-39 years suffered at least monthly leakage.

Melville and co-workers sent a 15-page self-report form to 6,000 women aged 30-90 years, who were enrolled in a large health 